\title{
Improved General Mapping Deformation Method for Nonlinear Partial Differential Equations in Mathematical Physics
}

\author{
Khaled A. Gepreel ${ }^{1,2}$ \\ ${ }^{1}$ Math Departement, Faculty of Science, Zagazig University, Zagazig, Egypt \\ ${ }^{2}$ Math Departement, Faculty of Science, Taif University, Taif, Saudi Arabia \\ Correspondence should be addressed to Khaled A. Gepreel; kagepreel@yahoo.com
}

Received 2 April 2013; Accepted 10 May 2013

Academic Editor: Abdel-Maksoud A. Soliman

Copyright (c) 2013 Khaled A. Gepreel. This is an open access article distributed under the Creative Commons Attribution License, which permits unrestricted use, distribution, and reproduction in any medium, provided the original work is properly cited.

\begin{abstract}
We use the improved general mapping deformation method based on the generalized Jacobi elliptic functions expansion method to construct some of the generalized Jacobi elliptic solutions for some nonlinear partial differential equations in mathematical physics via the generalized nonlinear Klein-Gordon equation and the classical Boussinesq equations. As a result, some new generalized Jacobi elliptic function-like solutions are obtained by using this method. This method is more powerful to find the exact solutions for nonlinear partial differential equations.
\end{abstract}

\section{Introduction}

The nonlinear partial differential equations play an important role to study many problems in physics and geometry. The effort in finding exact solutions to nonlinear equations is important for the understanding of most nonlinear physical phenomena. For instance, the nonlinear wave phenomena observed in fluid dynamics, plasma and optical fibers are often modeled by the bell-shaped sech solutions and the kinkshaped tanh solutions.

Many effective methods have been presented, such as inverse scattering transform method [1], Bäcklund transformation [2], Darboux transformation [3], Hirota bilinear method [4], variable separation approach [5], various tanh methods [6-9], homogeneous balance method [10], similarity reductions method [11, 12], $\left(G^{\prime} / G\right)$-expansion method [13], the reduction $\mathrm{mKdV}$ equation method [14], the trifunction method $[15,16]$, the projective Riccati equation method [17], the Weierstrass elliptic function method [18], the SineCosine method $[19,20]$, the Jacobi elliptic function expansion $[21,22]$, the complex hyperbolic function method [23], the truncated Painlevé expansion [24], the F-expansion method [25], the rank analysis method [26], the ansatz method [27, 28], the exp-function expansion method [29], and the subODE. method [30], Recently, Hong and Lü [31] put a good new method to obtain the exact solutions for the general variable coefficients $\mathrm{KdV}$ equation by using the improved general mapping deformation method based on the generalized Jacobi elliptic functions expansion method. In this paper, we use the improved general mapping deformation method based on the generalized Jacobi elliptic functions expansion method to obtain several new families of the exact solutions for some nonlinear partial differential equations such as the generalized Klein-Gordon equations and the classical Boussinesq equations which are very important in the mathematical physics.

\section{Description of the Improved General Mapping Deformation Method}

Suppose we have the following nonlinear PDE:

$$
F\left(u, u_{t}, u_{x}, u_{t t}, u_{x x}, u_{x t}, \ldots\right)=0
$$

where $u=u(x, t)$ is an unknown function, $F$ is a polynomial in $u=u(x, t)$, and its various partial derivatives in which the highest order derivatives and nonlinear terms are involved. In the following, we give the main steps of a deformation method. 
Step 1. The traveling wave variable

$$
u(x, t)=u(\xi), \quad \xi=x-V t,
$$

where $V$ is a constant, permits us reducing (1) to an ODE for $u=u(\xi)$ in the following form:

$$
P\left(u,-V u^{\prime}, u^{\prime}, V^{2} u^{\prime \prime},-V u^{\prime \prime}, u^{\prime \prime}, \ldots\right)=0,
$$

where $P$ is a polynomial of $u=u(\xi)$ and its total derivatives.

Step 2. Firstly, we assume that the solution equation (1) has the following form:

$$
u(\xi)=\sum_{i=0}^{N} A_{i} \phi^{i}(\xi)+\sum_{i=1}^{N} B_{i} \phi^{-i}(\xi),
$$

where $\xi=x-V t$ and $A_{i}, B_{i}(i=0,1,2, \ldots, N)$, and $V$ are arbitrary constants to be determined later, while $\phi(\xi)$ satisfies the following nonlinear first-order differential equation:

$$
\phi^{\prime 2}(\xi)=\sum_{i=0}^{4} a_{i} \phi^{i}(\xi) .
$$

Step 3. The positive integer " $N$ " can be determined by considering the homogeneous balance between the highest order partial derivative and nonlinear terms appearing in (1).

If " $N$ " is not positive integer. In order to apply this method when " $\mathrm{N}$ " is not positive integer (fraction or negative integer), we make the following transformations.

(1) When $N=p / q, q \neq 0$ is a fraction in lowest term, we take the following transformation:

$$
u(\xi)=Y^{p / q}(\xi)
$$

(2) When $N$ is negative integer, we take the following transformation:

$$
u(\xi)=Y^{-N}(\xi)
$$

and return to determine the value of $n$ again from the new equation. Therefore, we can get that the value of $n$ in (2) is positive integer.

Step 4. Substituting (4) into (3) with Condition (5), we obtain polynomial in $\phi^{j}(\xi) \phi^{\prime s}(\xi),(s=0,1 ; j=$ $\ldots,-2,-1,0,1,2, \ldots)$. Setting each coefficients of this polynomial to be zero yields a set of algebraic equations for $A_{i}, B_{i}(i=0,1,2, \ldots, N)$, and $V$.

Step 5. In order to obtain some new rational Jacobi elliptic solutions of (5), we assume that (5) has the following solutions:

$$
\phi(\xi)=c_{0}+c_{1} e(\xi)+c_{2} f(\xi)+c_{3} g(\xi)+c_{4} h(\xi),
$$

where $c_{i}=c_{i}(i=0, \ldots, 4)$ are arbitrary constants to be determined later, and the functions $e=e(\xi), f=f(\xi), g=$ $g(\xi), h=h(\xi)$ are expressed as follows [32, 33]:

$$
\begin{gathered}
e=\frac{1}{p+q \operatorname{sn}(\xi, m)+r c n(\xi, m)+l d n(\xi, m)}, \\
f=\frac{\operatorname{sn}(\xi, m)}{p+q \operatorname{sn}(\xi, m)+r c n(\xi, m)+l d n(\xi, m)}, \\
g=\frac{c n(\xi, m)}{p+q s n(\xi, m)+r c n(\xi, m)+l d n(\xi, m)}, \\
h=\frac{d n(\xi, m)}{p+q \operatorname{sn}(\xi, m)+r c n(\xi, m)+l d n(\xi, m)},
\end{gathered}
$$

where $p, q, r$, and $l$ are arbitrary constants and $s n(\xi, m)$, $c n(\xi, m)$, and $d n(\xi, m)$ are Jacobi elliptic functions with the modulus $m<1$.

Step 6. We substitute (8) and (9) into (5). Cleaning the denominator and collecting all terms with the same degree of $s n(\xi, m), c n(\xi, m)$, and $d n(\xi, m)$ together, the left-hand side of (5) is converted into a polynomial in $\operatorname{sn}(\xi, m), c n(\xi, m)$, and $d n(\xi, m)$. Setting each coefficients $\operatorname{sn}(\xi, m), c n(\xi, m)$, and $d n(\xi, m)$ of this polynomial to be zero, we derive a set of algebraic equations for $c_{i}(i=0, \ldots, 4), p, q, r$, and $l$.

Step 7. With the help of symbolic software package as Maple or Mathematica, we solve the system of algebraic equations which is obtained in Step 4 with the system obtained in Step 6 to calculate for $A_{i}, B_{i}(i=0,1,2, \ldots, N), V$, and $c_{i}(i=$ $0, \ldots, 4), p, q, r$, and $l$.

Step 8. Substituting the results in Step 7 into (4), (8), and (9), we will construct many new exact solutions for the nonlinear partial differential Equation (1).

\section{Application of the Improved General Mapping Deformation Method}

In this section, we use the proposed method to construct Jacobi elliptic traveling wave solutions for some nonlinear partial differential equations in mathematical physics, namely, the generalized nonlinear Klein-Gordon equation and the classical Boussinesq equations which are very important in the mathematical physics and have been paid attention by many researchers.

3.1. Example 1: The Generalized Nonlinear Klein-Gordon Equations. The generalized nonlinear Klein-Gordon equation [34] is in the following form:

$$
u_{t t}-k^{2} u_{x x}+\alpha u-\beta u^{1-n}+\gamma u^{n+1}=0, \quad n>2,
$$

where $k, \alpha, \beta$, and $\gamma$ are arbitrary constants. These equations play an important role in many scientific applications, such as the solid state physics, the nonlinear optics, and the quantum field theory (see $[17,18,24])$. Wazwaz $[19,35]$ investigated the nonlinear Klein-Gordon equations and found many types of 
exact traveling wave solutions including compact solutions, soliton solutions, solitary pattern solutions, and periodic solutions using the tanh-function method. The traveling wave variable (2) permits us to convert equation (10) into the following ODE:

$$
\left(V^{2}-k^{2}\right) u^{\prime \prime}+\alpha u-\beta u^{1-n}+\gamma u^{n+1}=0 .
$$

Considering the homogeneous balance between the highest order derivative $u^{\prime \prime}$ and the nonlinear term $u^{n+1}$ in (11), we get $N=2 / n$. According to Step 3, we use the following transformation:

$$
u=[\psi(\xi)]^{2 / n}
$$

where $\psi(\xi)$ is a new function of $\xi$. By substituting (12) into (11), we get the following new ODE:

$$
\begin{gathered}
\left(V^{2}-k^{2}\right)\left[2(2-n) \psi^{\prime 2}+2 n \psi \psi^{\prime \prime}\right] \\
+\alpha n^{2} \psi^{2}-\beta n^{2}+\gamma n^{2} \psi^{4}=0 .
\end{gathered}
$$

Determining the balance number $N$ of the new equation (13), we get $N=1$. Consequently, we have the formal solution of (13) in the following form:

$$
u(\xi)=A_{0}+A_{1} \phi(\xi)+B_{1} \phi^{-1}(\xi),
$$

where

$$
\phi^{\prime 2}(\xi)=\sum_{i=0}^{4} a_{i} \phi^{i}(\xi)
$$

We substitute (14) along with condition (15) into (13) and collect all terms with the same power of $\phi^{j}(\xi)\left[\phi^{\prime}(\xi)\right]^{s},(s=$ $0,1 ; j=\ldots,-2,-1,0,1,2, \ldots)$. Setting each coefficient of this polynomial to be zero, we get a system of the algebraic equations for $A_{0}, A_{1}, B_{1}, a_{0}, a_{1}, a_{2}, a_{3}, a_{4}$, and $V$. Also, we substitute (8) and (9) into (15). Cleaning the denominator and collecting all terms with the same degree of $\operatorname{sn}(\xi, m), c n(\xi, m)$, and $d n(\xi, m)$ together, the left-hand side of (15) is converted into a polynomial in $\operatorname{sn}(\xi, m), c n(\xi, m)$, and $d n(\xi, m)$. Setting each coefficients $s n(\xi, m), c n(\xi, m)$, and $d n(\xi, m)$ of these polynomials to be zero, we derive a system of the algebraic equations for $c_{i}=c_{i}(i=0, \ldots, 4), p, q, r$, and $l$.

With the help of Maple, we solve the system of the algebraic equations for $A_{0}, A_{1}, B_{1}, a_{0}, a_{1}, a_{2}, a_{3}, a_{4}$, and $V$ with the system algebraic equations for $c_{i}(i=0, \ldots, 4), p, q$, $r$, and $l$ to get the following results.

Case 1.

$$
\begin{aligned}
& A_{1}=\frac{p n}{c_{3}} \sqrt{\frac{2 \beta}{\left(V^{2}-K^{2}\right)\left(m^{2}-1\right)(n-2)},} \\
& \alpha=\frac{-2}{n^{2}}\left(V^{2}-K^{2}\right)\left(m^{2}+1\right), \\
& \gamma=\frac{-2}{4 \beta n^{4}}\left(V^{2}-K^{2}\right)^{2}\left(m^{2}-1\right)^{2}\left(n^{2}-4\right), \\
& a_{0}=\frac{-c_{3}^{2}\left(m^{2}-1\right)}{4 p^{2}}, \quad a_{2}=\frac{1+m^{2}}{2}, \\
& a_{4}=\frac{-p^{2}\left(m^{2}-1\right)}{4 c_{3}^{2}}, \quad q=p, \\
& A_{0}=B_{1}=a_{1}=a_{3}=l=r=c_{0} \\
& =c_{1}=c_{2}=c_{4}=0, \quad m \neq 1,
\end{aligned}
$$

where $p, \beta, V, c_{3}$, and $K$ are arbitrary constants. In this case, the rational Jacobi elliptic solution has the following form:

$$
\psi=\sqrt{\frac{2 n^{2} \beta}{\left(V^{2}-K^{2}\right)\left(m^{2}-1\right)(n-2)}} \frac{c n(\xi, m)}{[1+s n(\xi, m)]} .
$$

Consequently, the exact solution of the generalized nonlinear Klein-Gordon equation (10) takes the following form:

$$
u(x, t)=\left[\sqrt{\frac{2 n^{2} \beta}{\left(V^{2}-K^{2}\right)\left(m^{2}-1\right)(n-2)}} \frac{c n(\xi, m)}{[1+s n(\xi, m)]}\right]^{2 / n}
$$

where $\xi=x-t V$. In the special case when $m=0$, the trigonometric exact solution takes the following form:

$$
u(x, t)=\left[\sqrt{\frac{2 n^{2} \beta}{\left(K^{2}-V^{2}\right)(n-2)}} \frac{\cos (\xi)}{[1+\sin (\xi)]}\right]^{2 / n} .
$$


Case 2.

$$
\begin{aligned}
& A_{1}= \pm \frac{p n}{c_{3} m} \sqrt{\frac{\beta}{8\left(V^{2}-K^{2}\right)(n-2)}} \\
& B_{1}= \pm \frac{c_{3} n}{p m} \sqrt{\frac{\beta}{8\left(V^{2}-K^{2}\right)(n-2)}}, \\
& \alpha=\frac{-2}{n^{2}}\left(V^{2}-K^{2}\right)\left(2 m^{2}-1\right), \\
& \gamma=\frac{4 m^{2}}{\beta n^{4}}\left(V^{2}-K^{2}\right)^{2}\left(m^{2}-1\right)\left(n^{2}-4\right), \\
& a_{0}=\frac{-c_{3}^{2}\left(m^{2}-1\right)}{4 p^{2}}, \quad a_{2}=\frac{1+m^{2}}{2}, \\
& a_{4}=\frac{-p^{2}\left(m^{2}-1\right)}{4 c_{3}^{2}}, \quad q=p, m \neq 0 \\
& A_{0}=a_{1}=a_{3}=l=r=c_{0}=c_{1}=c_{2}=c_{4}=0,
\end{aligned}
$$

where $p, \beta, V, c_{3}$, and $K$ are arbitrary constants. In this case, the rational Jacobi elliptic solution has the following form:

$$
\begin{aligned}
\psi= & \pm \frac{n}{m} \sqrt{\frac{\beta}{8\left(V^{2}-K^{2}\right)(n-2)}} \frac{c n(\xi, m)}{[1 \pm s n(\xi, m)]} \pm \frac{n}{m} \\
& \times \sqrt{\frac{\beta}{8\left(V^{2}-K^{2}\right)(n-2)} \frac{[1 \pm s n(\xi, m)]}{c n(\xi, m)} .}
\end{aligned}
$$

Consequently, the exact solution of the generalized nonlinear Klein-Gordon equation (10) takes the following form:

$$
\begin{gathered}
u=\left[ \pm \frac{n}{m} \sqrt{\frac{\beta}{8\left(V^{2}-K^{2}\right)(n-2)}} \frac{c n(\xi, m)}{[1 \pm s n(\xi, m)]}\right. \\
\left.\quad \pm \frac{n}{m} \sqrt{\frac{\beta}{8\left(V^{2}-K^{2}\right)(n-2)}} \frac{[1 \pm s n(\xi, m)]}{c n(\xi, m)}\right]^{2 / n},
\end{gathered}
$$

where $\xi=x-V t$. In the special case when $m=1$, the hyperbolic exact solution takes the following form:

$$
\begin{gathered}
u=\left[ \pm n \sqrt{\frac{\beta}{8\left(V^{2}-K^{2}\right)(n-2)}} \frac{\operatorname{sech}(\xi)}{[1 \pm \tanh (\xi)]}\right. \\
\left.\quad \pm n \sqrt{\frac{\beta}{8\left(V^{2}-K^{2}\right)(n-2)}} \frac{[1 \pm \tanh (\xi)]}{\operatorname{sech}(\xi)}\right]^{2 / n} .
\end{gathered}
$$

Case 3.

$$
\begin{aligned}
& A_{1}= \pm q n \sqrt{\frac{\beta}{8\left(V^{2}-K^{2}\right)(n-2)\left(m^{2}-1\right)\left[\left(m^{2}-1\right) c_{3}^{2}-m^{2} c_{1}^{2}\right]}} \\
& B_{1}= \pm \frac{n}{q} \sqrt{\frac{\beta\left[\left(m^{2}-1\right) c_{3}^{2}-m^{2} c_{1}^{2}\right]}{8\left(V^{2}-K^{2}\right)(n-2)\left(m^{2}-1\right)}} \\
& \alpha=\frac{4}{n^{2}}\left(V^{2}-K^{2}\right)\left(m^{2}-2\right) \\
& \gamma=\frac{-4}{\beta n^{4}}\left(V^{2}-K^{2}\right)^{2}\left(m^{2}-1\right)\left(n^{2}-4\right) \\
& a_{0}=\frac{c_{1}^{2} m^{2}-\left(m^{2}-1\right) c_{3}^{2}}{4 q^{2}} \\
& A_{0}=a_{1}=\frac{1}{2}-a_{3}=m^{2}, \quad m \neq \pm 1 \\
& a_{4}=\frac{c_{3}^{2}-c_{1}^{2}}{4\left[c_{1}^{2} m^{2}-\left(m^{2}-1\right) c_{3}^{2}\right]} \\
& a_{2}^{2}\left(m^{2}-1\right) c_{3}^{2}
\end{aligned}
$$

where $V, \beta, c_{1}, c_{3}$, and $q$ are arbitrary constants. In this case, the rational Jacobi elliptic solution has the following form:

$$
\begin{aligned}
\psi= & \pm n \sqrt{\frac{\beta}{8\left(V^{2}-K^{2}\right)(n-2)\left(m^{2}-1\right)\left[\left(m^{2}-1\right) c_{3}^{2}-m^{2} c_{1}^{2}\right]}} \\
& \times \frac{\left(c_{1}+c_{3} c n(\xi, m)\right)}{\left[s n(\xi, m)+\sqrt{\left(c_{3}^{2}-c_{1}^{2}\right) /\left(m^{2}\left(c_{1}^{2}-c_{3}^{2}\right)+c_{3}^{2}\right)} d n(\xi, m)\right]} \\
& \pm n \sqrt{\frac{\beta\left[\left(m^{2}-1\right) c_{3}^{2}-m^{2} c_{1}^{2}\right]}{8\left(V^{2}-K^{2}\right)(n-2)\left(m^{2}-1\right)}} \\
& \times\left(\left[\operatorname{sn(\xi ,m)+\sqrt {\frac {c_{3}^{2}-c_{1}^{2}}{m^{2}(c_{1}^{2}-c_{3}^{2})+c_{3}^{2}}}} d n(\xi, m)\right]\right) \\
& \left.\times\left(c_{1}+c_{3} c n(\xi, m)\right)^{-1} \cdot\right]
\end{aligned}
$$


Consequently, the exact solution of the generalized nonlinear Klein-Gordon equation (10) takes the following form:

$$
\begin{aligned}
u=[ \pm & n \sqrt{\frac{\beta}{8\left(V^{2}-K^{2}\right)(n-2)\left(m^{2}-1\right)\left[\left(m^{2}-1\right) c_{3}^{2}-m^{2} c_{1}^{2}\right]}} \\
& \times\left(c_{1}+c_{3} c n(\xi, m)\right) \\
& \times\left[s n(\xi, m)+\sqrt{\frac{c_{3}^{2}-c_{1}^{2}}{m^{2}\left(c_{1}^{2}-c_{3}^{2}\right)+c_{3}^{2}}} d n(\xi, m)\right]^{-1} \\
& \pm \sqrt{\frac{\beta\left[\left(m^{2}-1\right) c_{3}^{2}-m^{2} c_{1}^{2}\right]}{8\left(V^{2}-K^{2}\right)(n-2)\left(m^{2}-1\right)}} \\
& \times\left[\operatorname{sn}(\xi, m)+\sqrt{\frac{c_{3}^{2}-c_{1}^{2}}{m^{2}\left(c_{1}^{2}-c_{3}^{2}\right)+c_{3}^{2}}} d n(\xi, m)\right] \\
& \left.\times\left(c_{1}+c_{3} c n(\xi, m)\right)^{-1}\right]^{2 / n},
\end{aligned}
$$

where $\xi=x-V t$. In the special case when $m=0$, the trigonometric exact solution takes the following form:

$$
\begin{aligned}
u=[ \pm & n \sqrt{\frac{\beta}{8\left(V^{2}-K^{2}\right)(n-2) c_{3}^{2}}}\left(c_{1}+c_{3} \cos (\xi)\right) \\
& \times\left[\sin (\xi)+\frac{1}{c_{3}} \sqrt{c_{3}^{2}-c_{1}^{2}}\right]^{-1} \pm n \sqrt{\frac{\beta c_{3}^{2}}{8\left(V^{2}-K^{2}\right)(n-2)}} \\
& \left.\times\left[\sin (\xi)+\frac{1}{c_{3}} \sqrt{c_{3}^{2}-c_{1}^{2}}\right]\left(c_{1}+c_{3} \cos (\xi)\right)^{-1}\right]^{2 / n} .
\end{aligned}
$$

Case 4.

$$
\begin{aligned}
& A_{0}= \pm \frac{n}{m} \sqrt{\frac{2 \beta}{\left(V^{2}-K^{2}\right)(n-2)},} \\
& A_{1}=\mp \frac{2 n r}{m c_{3}} \sqrt{\frac{2 \beta}{\left(V^{2}-K^{2}\right)(n-2)}}, \\
& \alpha=\frac{-2}{n^{2}}\left(V^{2}-K^{2}\right)\left(m^{2}-2\right), \\
& \gamma=\frac{m^{4}\left(V^{2}-K^{2}\right)^{2}\left(n^{2}-4\right)}{4 \beta n^{4}}, \\
& a_{0}=\frac{-c_{3}^{2}}{4 r^{2}}, \quad a_{1}=\frac{c_{3}}{r},
\end{aligned}
$$

$$
\begin{aligned}
& a_{2}=-1-m^{2}, \quad a_{3}=\frac{2 r m^{2}}{c_{3}}, \\
& a_{4}=\frac{-r^{2} m^{2}}{c_{3}^{2}}, \quad l= \pm i r, \\
& q= \pm r \sqrt{m^{2}-1}, \quad m \neq 0, \\
& B_{1}=p=c_{0}=c_{1}=c_{2}=c_{4}=0,
\end{aligned}
$$

where $V, \beta, K, c_{3}$, and $r$ are arbitrary constants and $i=$ $\sqrt{-1}$. In this case, the rational Jacobi elliptic solution has the following form:

$$
\begin{aligned}
\psi= & \pm \frac{n}{m} \sqrt{\frac{2 \beta}{\left(V^{2}-K^{2}\right)(n-2)}} \\
& \mp \frac{2 n}{m} \sqrt{\frac{2 \beta}{\left(V^{2}-K^{2}\right)(n-2)}} \\
& \times \frac{c n(\xi, m)}{\left[c n(\xi, m)+\sqrt{m^{2}-1} \operatorname{sn}(\xi, m) \pm i d n(\xi, m)\right]} .
\end{aligned}
$$

Consequently, the exact solution of the generalized nonlinear Klein-Gordon equation (10) takes the following form:

$$
\begin{aligned}
& u=\left[ \pm \frac{n}{m} \sqrt{\frac{2 \beta}{\left(V^{2}-K^{2}\right)(n-2)}}\right. \\
& \mp \frac{2 n}{m} \sqrt{\frac{2 \beta}{\left(V^{2}-K^{2}\right)(n-2)}} \\
& \left.\times \frac{c n(\xi, m)}{\left[c n(\xi, m)+\sqrt{m^{2}-1} \operatorname{sn}(\xi, m) \pm i d n(\xi, m)\right]}\right]^{2 / n},
\end{aligned}
$$

where $\xi=x-V t$. There are many cases that are omitted for convenience.

3.2. Example 2: The Classical Boussinesq Equations. In this subsection, we consider the classical Boussinesq equations [36] in the following form:

$$
\begin{aligned}
& v_{t}+[(1+v) u]_{x}+\frac{1}{3} u_{x x x}=0, \\
& u_{t}+u u_{x}+v_{x}=0 .
\end{aligned}
$$

The system (31) is integrable and has three Hamiltonian structures [36]. Wu and Zhang [37] derive three sets of classical Boussinesq model equations for modeling nonlinear and dispersive long gravity wave traveling in two horizontal directions on shallow water of uniform depth. This system is considered to be one of them. 
Let us now solve (31) by the proposed method. To this end, we see that the traveling wave variables $u=u(\xi), v=v(\xi)$, and $\xi=x-V t$, permit us to convert (31) into the following ODEs:

$$
\begin{aligned}
& C_{1}-V v+(1+v) u+\frac{1}{3} u^{\prime \prime}=0, \\
& C_{2}-V u+\frac{1}{2} u^{2}+v=0,
\end{aligned}
$$

where $C_{1}$ and $C_{2}$ are the integration constants. Considering the homogeneous balance between the highest order derivative and the nonlinear terms in (32), we get $M_{1}=1$ and $M_{2}=2$. Thus, the solutions of (32) have the following forms:

$$
\begin{aligned}
u(\xi)= & A_{0}+A_{1} \phi(\xi)+A_{2} \phi^{-1}(\xi), \\
v(\xi)= & B_{0}+B_{1} \phi(\xi)+B_{2} \phi^{2}(\xi) \\
& +B_{3} \phi^{-1}(\xi)+B_{4} \phi^{-2}(\xi),
\end{aligned}
$$

where

$$
\phi^{\prime 2}(\xi)=\sum_{i=0}^{4} a_{i} \phi^{i}(\xi)
$$

We substitute (33) along with Condition (34) into (32) and collect all terms with the same power of $\phi^{j}(\xi)\left[\phi^{\prime}(\xi)\right]^{s},(s=$ $0,1 ; j=\ldots,-2,-1,0,1,2, \ldots)$. Setting each coefficient of this polynomial to be zero, we get a system of the algebraic equations for $A_{0}, A_{1}, A_{2}, B_{0}, B_{1}, B_{2}, B_{3}, B_{4}, a_{0}, a_{1}, a_{2}, a_{3}, a_{4}$, and $V$. Also, we substitute (8) and (9) into (34). Cleaning the denominator and collecting all terms with the same degree of $\operatorname{sn}(\xi, m), c n(\xi, m)$, and $d n(\xi, m)$ together, the left-hand side of (34) is converted into a polynomial in $\operatorname{sn}(\xi, m), c n(\xi, m)$, and $d n(\xi, m)$. Setting each coefficients $\operatorname{sn}(\xi, m), c n(\xi, m)$, and $d n(\xi, m)$ of this polynomial to be zero, we derive a system of the algebraic equations for $c_{i}(i=0, \ldots, 4), p, q, r$, and $l$. With the help of Maple, we solve the system of the algebraic equations for $A_{0}, A_{1}, B_{1}, a_{0}, a_{1}, a_{2}, a_{3}, a_{4}$, and $V$ with the system algebraic equations for $c_{i}(i=0, \ldots, 4), p, q, r$ and $l$ to get the following results.

Case 1.

$$
\begin{aligned}
& A_{0}=-V, \quad A_{1}= \pm \frac{q \sqrt{1-m^{2}}}{\sqrt{3} c_{3}}, \\
& B_{0}=\frac{-\left(7+m^{2}\right)}{6}, \quad B_{2}=\frac{q^{2}\left(m^{2}-1\right)}{6 c_{3}^{2}}, \\
& C_{2}=\frac{1}{6}\left(3 V^{2}+7+m^{2}\right), \quad C_{1}=V, \\
& p= \pm q, \quad a_{0}=\frac{-c_{3}^{2}\left(m^{2}-1\right)}{4 q^{2}}, \\
& a_{2}=\frac{1+m^{2}}{2}, \quad a_{4}=\frac{\mp q^{2}\left(m^{2}-1\right)}{4 c_{3}^{2}}, \\
& A_{2}=B_{1}=B_{3}=B_{4}=c_{0}=c_{1}=c_{2}=c_{4}=0,
\end{aligned}
$$

where $q, V$, and $c_{3}$ are arbitrary constants. In this case, the rational Jacobi elliptic solution has the following form:

$$
\begin{gathered}
u=-V \pm \frac{q \sqrt{1-m^{2}}}{\sqrt{3} c_{3}} \frac{c n(\xi, m)}{[1 \pm \operatorname{sn}(\xi, m)]} \\
v=\frac{-\left(7+m^{2}\right)}{6}+\frac{\left(m^{2}-1\right)}{6} \frac{c n^{2}(\xi, m)}{[1 \pm \operatorname{sn}(\xi, m)]^{2}}
\end{gathered}
$$

where $\xi=x-V t$. In the special case when $m=0$, the trigonometric exact solution takes the following form:

$$
\begin{aligned}
& u=-V \pm \frac{q}{\sqrt{3} c_{3}} \frac{\cos (\xi)}{[1 \pm \sin (\xi)]} \\
& v=\frac{-7}{6}+\frac{-1}{6} \frac{\cos ^{2}(\xi)}{[1 \pm \sin (\xi)]^{2}} .
\end{aligned}
$$

Case 2.

$$
\begin{aligned}
& A_{0}=-V, \quad A_{1}= \pm \frac{q \sqrt{1-m^{2}}}{\sqrt{3} c_{3}} \\
& A_{2}= \pm \frac{c_{3} \sqrt{1-m^{2}}}{\sqrt{3} q}, \quad B_{0}=\frac{-\left(m^{2}+2\right)}{3}, \\
& B_{2}=\frac{q^{2}\left(m^{2}-1\right)}{6 c_{3}^{2}}, \quad B_{4}=\frac{c_{3}^{2}\left(m^{2}-1\right)}{6 q^{2}}, \\
& C_{2}=\frac{1}{6}\left(3 V^{2}+4+4 m^{2}\right), \quad C_{1}=V \\
& p= \pm q, \quad m \neq \pm 1, \quad a_{0}=\frac{-c_{3}^{2}\left(m^{2}-1\right)}{4 q^{2}} \\
& a_{2}=\frac{1+m^{2}}{2}, \quad a_{4}=\frac{-q^{2}\left(m^{2}-1\right)}{4 c_{3}^{2}} \\
& B_{1}=B_{3}=c_{0}=c_{1}=c_{2}=c_{4}=0,
\end{aligned}
$$

where $q, V$, and $c_{3}$, are arbitrary constants. In this case, the rational Jacobi elliptic solution has the following form:

$$
\begin{aligned}
u= & -V \pm \frac{\sqrt{1-m^{2}}}{\sqrt{3}} \frac{c n(\xi, m)}{[1 \pm s n(\xi, m)]} \\
& \pm \frac{\sqrt{1-m^{2}}}{\sqrt{3}} \frac{[1 \pm s n(\xi, m)]}{c n(\xi, m)}, \\
v= & \frac{-\left(7+m^{2}\right)}{6}+\frac{\left(m^{2}-1\right)}{6} \frac{c n^{2}(\xi, m)}{[1 \pm s n(\xi, m)]^{2}} \\
& +\frac{\left(m^{2}-1\right)}{6} \frac{[1 \pm s n(\xi, m)]^{2}}{c n^{2}(\xi, m)},
\end{aligned}
$$


where $\xi=x-V t$. In the special case when $m=0$, the trigonometric exact solution takes the following form:

$$
\begin{aligned}
& u=-V \pm \frac{1}{\sqrt{3}} \frac{\cos (\xi)}{[1 \pm \sin (\xi)]} \pm \frac{1}{\sqrt{3}} \frac{[1 \pm \sin (\xi)]}{\cos (\xi, m)} \\
& v=\frac{-7}{6}-\frac{1}{6} \frac{\cos ^{2}(\xi)}{[1 \pm \sin (\xi)]^{2}}-\frac{1}{6} \frac{[1 \pm \sin (\xi)]^{2}}{\cos ^{2}(\xi)} .
\end{aligned}
$$

Case 3.

$$
\begin{aligned}
& A_{0}=-V, \quad A_{1}= \pm \frac{q}{\sqrt{3} m c_{1}}, \\
& A_{2}= \pm \frac{m c_{1}}{\sqrt{3} q}, \quad B_{0}=\frac{3+m^{2}}{3}, \\
& B_{2}=-\frac{q^{2}}{6 m^{2} c_{1}^{2}}, \quad B_{4}=-\frac{c_{1}^{2} m^{2}}{6 q^{2}}, \\
& C_{2}=\frac{1}{6}\left(3 V^{2}+4-2 m^{2}\right), \quad C_{1}=V, \\
& a_{0}=\frac{m^{2} c_{1}^{2}}{4 q^{2}}, \quad a_{2}=\frac{1}{2}-m^{2}, \\
& a_{4}=\frac{q^{2}}{4 m^{2} c_{1}^{2}}, \quad l=\frac{ \pm i q}{m}, \quad m \neq 0, \\
& B_{1}=B_{3}=a_{1}=a_{3}=p=r=c_{0}=c_{3}=c_{2}=c_{4}=0,
\end{aligned}
$$

where $q, V$, and $c_{1}$ are arbitrary constants. In this case, the rational Jacobi elliptic solution has the following form:

$$
\begin{aligned}
u= & -V \pm \frac{1}{\sqrt{3}[m \operatorname{sn}(\xi, m) \pm i d n(\xi, m)]} \\
& \pm \frac{[m \operatorname{sn}(\xi, m) \pm i d n(\xi, m)]}{\sqrt{3}}, \\
v= & \frac{3+m^{2}}{3}-\frac{1}{6[m \operatorname{sn}(\xi, m) \pm i d n(\xi, m)]^{2}} \\
& -\frac{[m \operatorname{sn}(\xi, m) \pm i d n(\xi, m)]^{2}}{6},
\end{aligned}
$$

where $\xi=x-V t$. In the special case when $m=1$, the hyperbolic exact solution takes the following form:

$$
\begin{aligned}
u= & -V \pm \frac{1}{\sqrt{3}[\tanh (\xi) \pm i \operatorname{sech}(\xi)]} \\
& \pm \frac{1}{\sqrt{3}}[\tanh (\xi) \pm i \operatorname{sech}(\xi)] \\
v= & \frac{4}{3}-\frac{1}{6[\tanh (\xi) \pm i \operatorname{sech}(\xi)]^{2}} \\
- & \frac{1}{6}[\tanh (\xi) \pm i \operatorname{sech}(\xi)]^{2} .
\end{aligned}
$$

Case 4.

$$
\begin{aligned}
& A_{0}=-V, \quad A_{1}= \pm \frac{q}{\sqrt{3\left[c_{1}^{2} m^{2}-\left(m^{2}-1\right) c_{3}^{2}\right]}}, \\
& B_{0}=\frac{m^{2}-3}{3}, \quad A_{2}= \pm \frac{\sqrt{\left[c_{1}^{2} m^{2}-\left(m^{2}-1\right) c_{3}^{2}\right]}}{\sqrt{3} q}, \\
& B_{2}=-\frac{q^{2}}{6\left[c_{1}^{2} m^{2}-\left(m^{2}-1\right) c_{3}^{2}\right]}, \\
& B_{4}=-\frac{\left[c_{1}^{2} m^{2}-\left(m^{2}-1\right) c_{3}^{2}\right]}{6 q^{2}}, \\
& a_{0}=\frac{c_{1}^{2} m^{2}-\left(m^{2}-1\right) c_{3}^{2}}{4 q^{2}}, \quad a_{2}=\frac{1}{2}-m^{2}, \\
& a_{4}=\frac{q^{2}}{4\left[c_{1}^{2} m^{2}-\left(m^{2}-1\right) c_{3}^{2}\right]}, \\
& l=q \sqrt{\frac{c_{3}^{2}-c_{1}^{2}}{m^{2} c_{1}^{2}-\left(m^{2}-1\right) c_{3}^{2}},} \\
& B_{3}=B_{1}=a_{1}=a_{3}=p=r=c_{0}=c_{2}=c_{4}=0,
\end{aligned}
$$

where $p, \beta, \alpha, c_{1}$, and $c_{3}$ are arbitrary constants. In this case, the rational Jacobi elliptic solution has the following form:

$$
\begin{aligned}
u= & -V \pm\left(c_{1}+c_{3} c n(\xi, m)\right) \\
\times & \left(\sqrt{3\left[c_{1}^{2} m^{2}-\left(m^{2}-1\right) c_{3}^{2}\right]}\right. \\
& \left.\times\left[s n(\xi, m)+\sqrt{\frac{c_{3}^{2}-c_{1}^{2}}{m^{2} c_{1}^{2}-\left(m^{2}-1\right) c_{3}^{2}}} d n(\xi, m)\right]\right)^{-1} \\
\pm & \left(\sqrt{\left[c_{1}^{2} m^{2}-\left(m^{2}-1\right) c_{3}^{2}\right]}\right. \\
& \left.\times\left[s n(\xi, m)+\sqrt{\frac{c_{3}^{2}-c_{1}^{2}}{m^{2} c_{1}^{2}-\left(m^{2}-1\right) c_{3}^{2}}} d n(\xi, m)\right]\right)^{-1} \\
& \times\left(\sqrt{3}\left\{c_{1}+c_{3} c n(\xi, m)\right\}\right)^{-1}, \\
v= & \frac{m^{2}-3}{3}-\left(\left(c_{1}+c_{3} c n(\xi, m)\right)^{2}\right)
\end{aligned}
$$




$$
\begin{aligned}
& \times\left(6\left[c_{1}^{2} m^{2}-\left(m^{2}-1\right) c_{3}^{2}\right]\right. \\
& \left.\times\left[s n(\xi, m)+\sqrt{\frac{c_{3}^{2}-c_{1}^{2}}{m^{2} c_{1}^{2}-\left(m^{2}-1\right) c_{3}^{2}}} d n(\xi, m)\right]^{2}\right)^{-1} \\
& -\left(\left[c_{1}^{2} m^{2}-\left(m^{2}-1\right) c_{3}^{2}\right]\right. \\
& \left.\times\left[\operatorname{sn}(\xi, m)+\sqrt{\frac{c_{3}^{2}-c_{1}^{2}}{m^{2} c_{1}^{2}-\left(m^{2}-1\right) c_{3}^{2}}} d n(\xi, m)\right]^{2}\right) \\
& \times\left(6\left(c_{1}+c_{3} c n(\xi, m)\right)^{2}\right)^{-1},
\end{aligned}
$$

where $\xi=x-V t$. In the special case when $m=1$, the hyperbolic exact solution takes the following form:

$$
\begin{aligned}
u & =-V \pm \frac{c_{1}+c_{3} \operatorname{sech}(\xi)}{\sqrt{3}\left[c_{1} \tanh (\xi)+\sqrt{c_{3}^{2}-c_{1}^{2}} \operatorname{sech}(\xi)\right]} \\
& \pm \frac{\left[c_{1} \tanh (\xi)+\sqrt{c_{3}^{2}-c_{1}^{2}} \operatorname{sech}(\xi)\right]}{\sqrt{3}\left\{c_{1}+c_{3} \operatorname{sech}(\xi)\right\}}, \\
v & =\frac{-2}{3}-\frac{\left(c_{1}+c_{3} \operatorname{sech}(\xi)\right)^{2}}{6\left[c_{1} \tanh (\xi)+\sqrt{c_{3}^{2}-c_{1}^{2}} \operatorname{sech}(\xi)\right]^{2}} \\
& -\frac{\left[c_{1} \tanh (\xi)+\sqrt{c_{3}^{2}-c_{1}^{2}} \operatorname{sech}(\xi)\right]^{2}}{6\left(c_{1}+c_{3} \operatorname{sech}(\xi)\right)^{2}} .
\end{aligned}
$$

Also, in the special case when $m=0$, the trigonometric exact solution takes the following form:

$$
\begin{aligned}
u= & -V \pm \frac{c_{1}+c_{3} \cos (\xi)}{\sqrt{3}\left[c_{3} \sin (\xi)+\sqrt{c_{3}^{2}-c_{1}^{2}}\right]} \\
& \pm \frac{\left[c_{3} \sin (\xi)+\sqrt{c_{3}^{2}-c_{1}^{2}}\right]}{\sqrt{3}\left\{c_{1}+c_{3} \cos (\xi)\right\}}, \\
v= & -1-\frac{\left(c_{1}+c_{3} \cos (\xi)\right)^{2}}{6\left[c_{3} \sin (\xi)+\sqrt{c_{3}^{2}-c_{1}^{2}}\right]^{2}} \\
& -\frac{\left[c_{3} \sin (\xi)+\sqrt{c_{3}^{2}-c_{1}^{2}}\right]^{2}}{6\left(c_{1}+c_{3} \cos (\xi)\right)^{2}} .
\end{aligned}
$$

\section{Conclusion}

In this paper, the improved general mapping deformation method based on the generalized Jacobi elliptic functions expansion method with computerized symbolic computation is used to construct the exact solutions for some nonlinear partial differential equations in mathematical physics when the balance number is positive integer or not positive integer. This method allowed us to calculate many new exact solutions for nonlinear partial differential equations in mathematical physics. The Jacobi elliptic solutions that are obtained by this method are the generalization to the hyperbolic function solutions and trigonometric function solutions when the modulus $m \rightarrow 1$ and $m \rightarrow 0$. This method is reliable and concise and gives more exact solutions compared to the other methods.

\section{Appendix}

The Jacobi elliptic functions $s n(\xi, m), c n(\xi, m)$, and $d n(\xi, m)$ generate into hyperbolic functions when $m \rightarrow 1$ as follows $[38,39]$ :

$$
\begin{gathered}
\operatorname{sn}(\xi, m) \longrightarrow \tanh (x), \quad c n(\xi, m) \longrightarrow \operatorname{sech}(\xi), \\
d n(\xi, m) \longrightarrow \operatorname{sech}(\xi),
\end{gathered}
$$

and into trigonometric functions when $m \rightarrow 0$ as follows:

$$
\begin{gathered}
\operatorname{sn}(\xi, m) \longrightarrow \sin (\xi), \quad c n(\xi, m) \longrightarrow \cos (\xi), \\
d n(\xi, m) \longrightarrow 1 .
\end{gathered}
$$

\section{Acknowledgment}

The author thanks the referees for their helpful suggestions that improved the content of the paper.

\section{References}

[1] M. J. Ablowitz and P. A. Clarkson, Soliton Nonlinear Evolution Equations and Inverse Scattering, Cambridge University Press, New York, NY, USA, 1991.

[2] C. H. Gu, Soliton Theory and Its Application, Zhejiang Science and Technology Press, 1990.

[3] V. B. Matveev and M. A. Salle, Darboux Transformation and Soliton, Springer, Berlin, Germany, 1991.

[4] R. Hirota, The Direct Method in Soliton Theory, Cambridge University Press, Cambridge, UK, 2004.

[5] S.-Y. Lou and J. Z. Lu, "Special solutions from the variable separation approach: the Davey-Stewartson equation," Journal of Physics A, vol. 29, no. 14, pp. 4209-4215, 1996.

[6] E. J. Parkes and B. R. Duffy, "Travelling solitary wave solutions to a compound KdV-Burgers equation," Physics Letters A, vol. 229, no. 4, pp. 217-220, 1997.

[7] E. Fan, "Extended tanh-function method and its applications to nonlinear equations," Physics Letters A, vol. 277, no. 4-5, pp. 212-218, 2000.

[8] Z. Y. Yan, "New explicit travelling wave solutions for two new integrable coupled nonlinear evolution equations," Physics Letters A, vol. 292, no. 1-2, pp. 100-106, 2001. 
[9] Y. Chen and Y. Zheng, "Generalized extended tanh-function method to construct new explicit exact solutions for the approximate equations for long water waves," International Journal of Modern Physics C, vol. 14, no. 5, pp. 601-611, 2003.

[10] M. L. Wang, Y. Zhou, and Z. Li, "Application of a homogeneous balance method to exact solutions of nonlinear equations in mathematical physics," Physics Letters A, vol. 216, no. 1-5, pp. 67-75, 1996.

[11] G. W. Bluman and S. Kumei, Symmetries and Differential Equations, Springer, New York, NY, USA, 1989.

[12] P. J. Olver, Applications of Lie Groups to Differential Equations, Springer, New York, NY, USA, 1986.

[13] E. M. E. Zayed and K. A. Gepreel, “The (G'/G-) expansion method for finding traveling wave solutions of nonlinear partial differential equations in mathematical physics," Journal of Mathematical Physics, vol. 50, no. 1, Article ID 013502, 12 pages, 2009.

[14] Z. Y. Yan, "A reduction mKdV method with symbolic computation to constract new doubly-periodic solutions for nonlinear wave equations," International Journal of Modern Physics $C$, vol. 14, pp. 661-672, 2003.

[15] Z. Y. Yan, "The new tri-function method to multiple exact solutions of nonlinear wave equations," Physica Scripta, vol. 78, no. 3, Article ID 035001, 2008.

[16] Z. Y. Yan, "Periodic, solitary and rational wave solutions of the 3D extended quantum Zakharov-Kuznetsov equation in dense quantum plasmas," Physics Letters A, vol. 373, no. 29, pp. 24322437, 2009.

[17] D. C. Lu and B. J. Hong, "New exact solutions for the $(2+1)$ dimensional generalized Broer-Kaup system," Applied Mathematics and Computation, vol. 199, no. 2, pp. 572-580, 2008.

[18] A. V. Porubov, "Periodical solution to the nonlinear dissipative equation for surface waves in a convecting liquid layer," Physics Letters A, vol. 221, no. 6, pp. 391-394, 1996.

[19] A.-M. Wazwaz, "The tanh and the sine-cosine methods for compact and noncompact solutions of the nonlinear KleinGordon equation," Applied Mathematics and Computation, vol. 167, no. 2, pp. 1179-1195, 2005.

[20] Z. Y. Yan and H. Q. Zhang, "New explicit solitary wave solutions and periodic wave solutions for Whitham-Broer-Kaup equation in shallow water," Physics Letters A, vol. 285, no. 5-6, pp. 355362, 2001.

[21] D. A. Lü, "Jacobi elliptic function solutions for two variant Boussinesq equations," Chaos, Solitons and Fractals, vol. 24, no. 5, pp. 1373-1385, 2005.

[22] Z. Y. Yan, "Abundant families of Jacobi elliptic function solutions of the $(2+1)$-dimensional integrable Davey-Stewartsontype equation via a new method," Chaos, Solitons and Fractals, vol. 18, no. 2, pp. 299-309, 2003.

[23] C. L. Bai and H. Zhao, "Generalized method to construct the solitonic solutions to $(3+1)$-dimensional nonlinear equation," Physics Letters A, vol. 354, no. 5-6, pp. 428-436, 2006.

[24] F. Cariello and M. Tabor, "Similarity reductions from extended Painlevé expansions for nonintegrable evolution equations," Physica D, vol. 53, no. 1, pp. 59-70, 1991.

[25] M. Wang and X. Li, "Extended F-expansion method and periodic wave solutions for the generalized Zakharov equations," Physics Letters A, vol. 343, no. 1-3, pp. 48-54, 2005.

[26] X. Feng, "Exploratory approach to explicit solution of nonlinear evolution equations," International Journal of Theoretical Physics, vol. 39, no. 1, pp. 207-222, 2000.
[27] J. L. Hu, "Explicit solutions to three nonlinear physical models," Physics Letters A, vol. 287, no. 1-2, pp. 81-89, 2001.

[28] J. L. Hu and H. Zhang, "A new method for finding exact traveling wave solutions to nonlinear partial differential equations," Physics Letters A, vol. 286, no. 2-3, pp. 175-179, 2001.

[29] J.-H. He and X.-H. Wu, "Exp-function method for nonlinear wave equations," Chaos, Solitons \& Fractals, vol. 30, no. 3, pp. 700-708, 2006.

[30] X. Z. Li and M. L. Wang, "A sub-ODE method for finding exact solutions of a generalized $\mathrm{KdV}-\mathrm{mKdV}$ equation with high-order nonlinear terms," Physics Letters A, vol. 361, no. 1-2, pp. 115-118, 2007.

[31] B. J. Hong and D. Lü, "New Jacobi elliptic function-like solutions for the general KdV equation with variable coefficients," Mathematical and Computer Modelling, vol. 55, no. 3-4, pp. 1594-1600, 2012.

[32] B. J. Hong, "New Jacobi elliptic functions solutions for the variable-coefficient MKdV equation," Applied Mathematics and Computation, vol. 215, no. 8, pp. 2908-2913, 2009.

[33] B. J. Hong, "New exact Jacobi elliptic functions solutions for the generalized coupled Hirota-Satsuma KdV system," Applied Mathematics and Computation, vol. 217, no. 2, pp. 472-479, 2010.

[34] K. A. Gepreel, "Exact solutions for nonlinear PDEs with the variable coefficients in mathematical physics," Journal of Computational Science, vol. 6, no. 1, pp. 3-14, 2011.

[35] A.-M. Wazwaz, "New solitary wave solutions to the modified Kawahara equation," Physics Letters A, vol. 360, no. 4-5, pp. 588592, 2007.

[36] B. A. Kupershmidt, "Mathematics of dispersive water waves," Communications in Mathematical Physics, vol. 99, no. 1, pp. 5173, 1985.

[37] T. Y. Wu and J. E. Zhang, “On modeling nonlinear long waves," in Mathematics is for Solving Problems, V. Roytburd, L. Pamela Cook, and M. Tulin, Eds., pp. 233-241, SIAM, Philadelphia, Pa, USA, 1996.

[38] K. A. Gepreel, "A generalized $\left(G^{\prime} / G\right)$-expansion method to find the traveling wave solutions of nonlinear evolution equations," Journal of Partial Differential Equations, vol. 24, no. 1, pp. 55-69, 2011.

[39] K. A. Gepreel and A. R. Shehata, "Jacobi elliptic solutions for nonlinear differential difference equations in mathematical physics," Journal of Applied Mathematics, vol. 2012, Article ID 710375, 15 pages, 2012. 


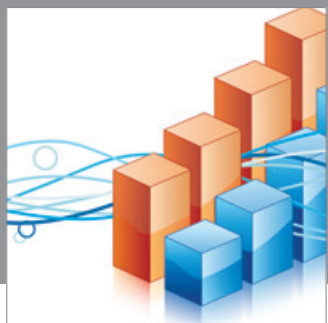

Advances in

Operations Research

mansans

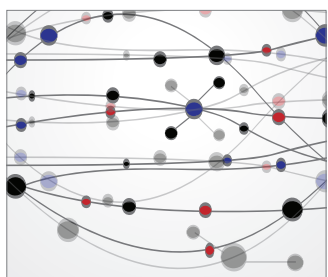

The Scientific World Journal
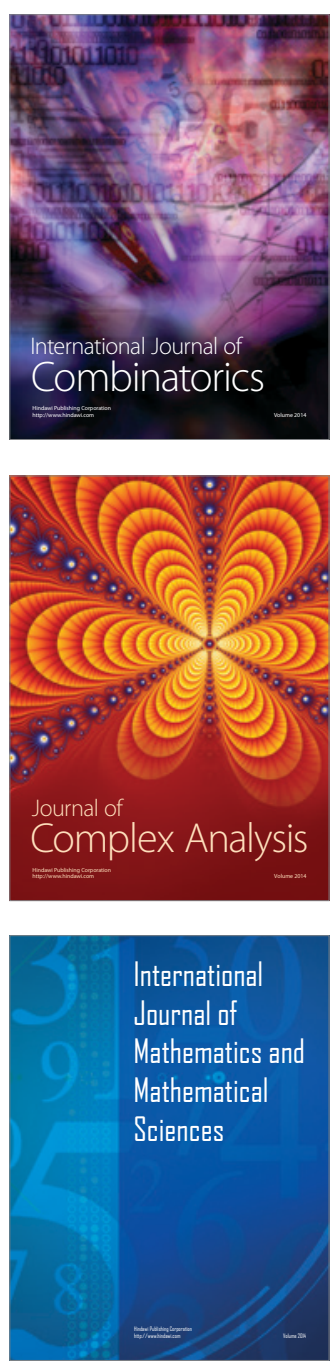
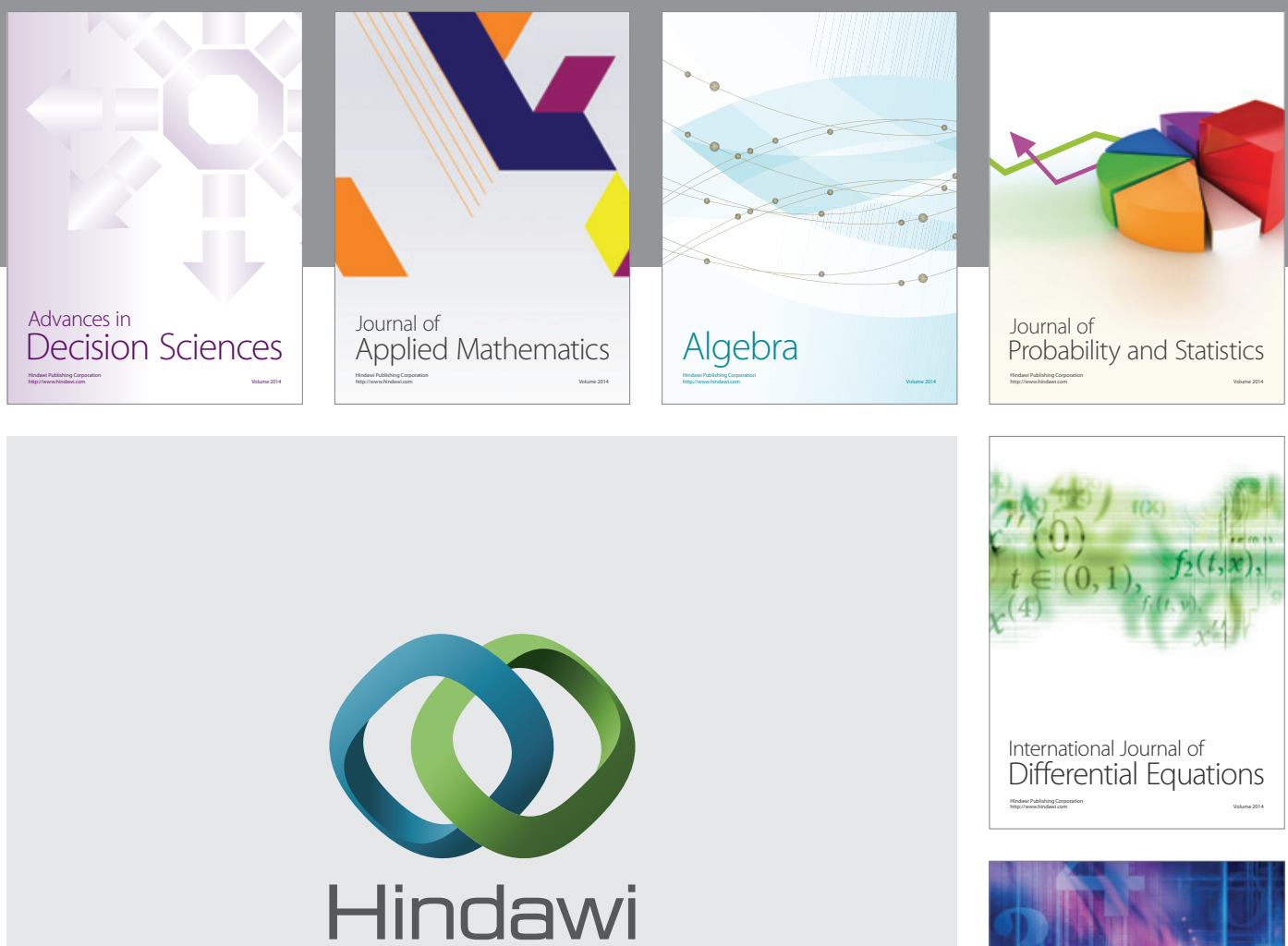

Submit your manuscripts at http://www.hindawi.com
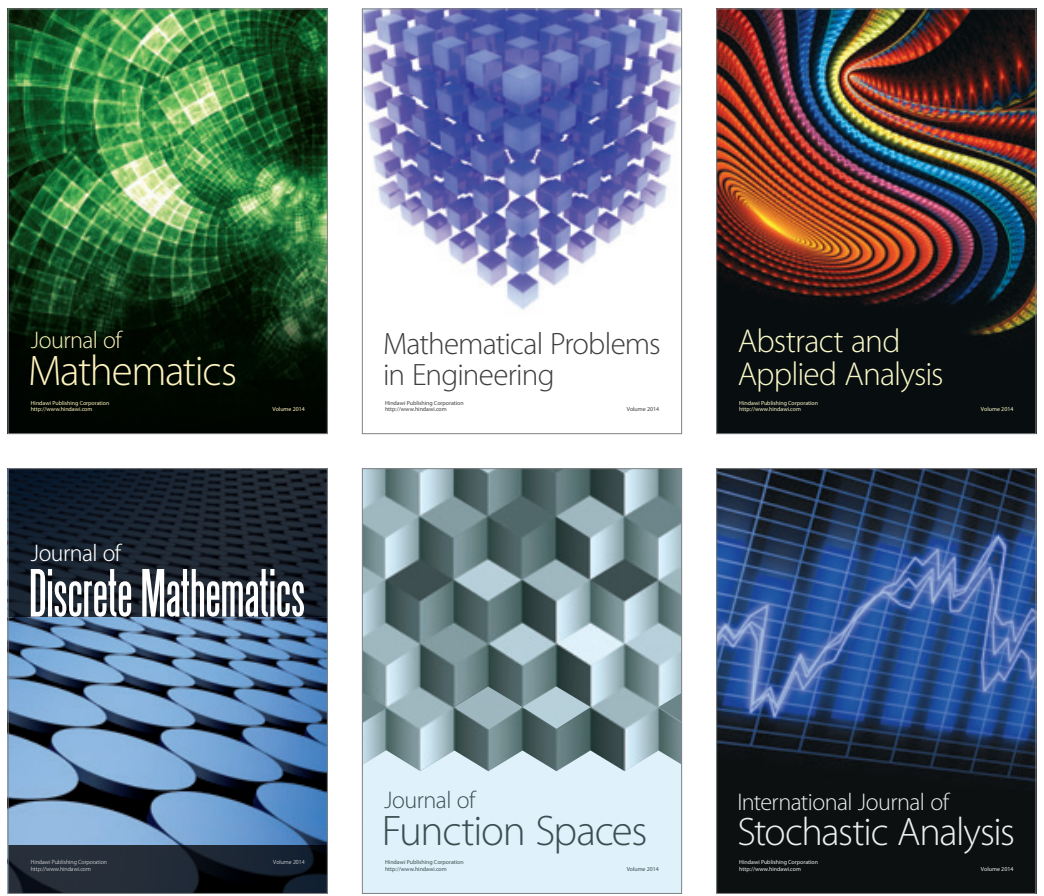

Journal of

Function Spaces

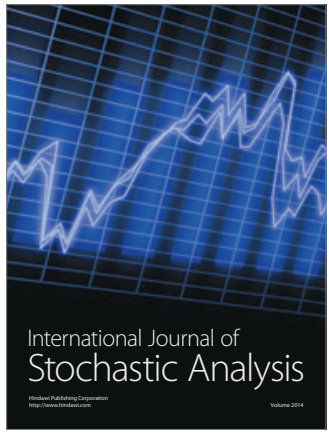

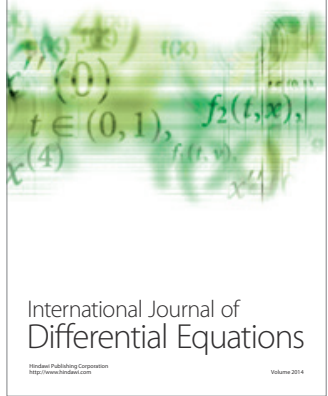
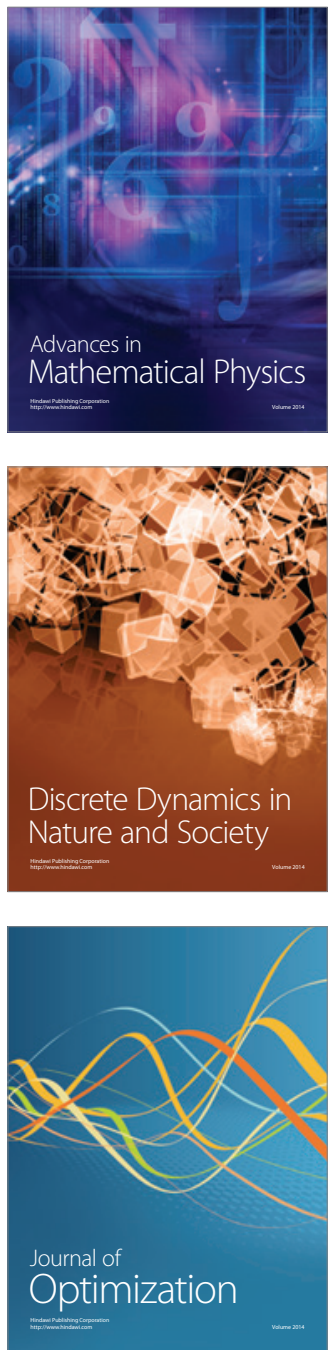